\title{
Nose to Brain Drug Delivery: A Recent Update
}

\section{Chatterjee B*}

Department of Pharmaceutical Technology, Kulliyyyah of Pharmacy, International Islamic University Malaysia, Kuantan, Malayisa

\begin{abstract}
Drugs used to treat brain or central nervous system disorder suffer from shortfall of absorption and reaching brain due to blood brain barrier and cerebro spinal fluid barrier. Intra nasal administration of brain targeted drug delivery system is gaining popularity to circumvent blood brain barrier and enhance drug availability in brain. It also shows promising reduction of systemic adverse effect incurred by the drug. However there are major limitation of this route which restrict its success of application. Despite different dosage forms such as micro or nano emulsion, gel, in situ gel etc the intra nasal route is still lacking wide applicability. This short review aims to highlight some important features related to nose to brain drug delivery along with its potential challenges that need to overcome. It has focused on few interesting or significant articles on the matter published within last 4-5 years.
\end{abstract}

Keywords: Nose to brain; In situ gel; Intra nasal; Blood brain barrier; Nano emulsion

\section{Introduction}

\section{Reaching brain or CNS is a potential challenge}

The unique structure of brain relative to other organs of human body is the outer liquid cover called cerebrospinal fluid (CSF) and inner microvascular layer called blood brain barrier (BBB) [1]. These two barriers form a sophisticated protection system for brain to preserve its physiological nature and shield from external threats such as toxins or pathogens. The BBB forms the interface between blood capillaries and brain tissue. It has its own unique nature which allows selective access of necessary nutrients and hormones while restricts entry of other external materials including therapeutic agent or drug [2]. Any drug that needs to reach central nervous system (CNS) requires crossing BBB. Almost all large drug molecules and most of the small molecules cannot enter brain or CNS after oral or systemic administration. Active transport and passive diffusion through endothelial cells are the two principal mechanisms by which molecules can enter the brain. But problem is that the endothelial cells of capillaries present in the BBB are effectively precluded by very stringent and tight junctions [3]. Therefore other than modified form of drug delivery especially designed for CNS, normal oral or parenteral route of drug administration is not capable enough to make the drug available at the site. This problem is much higher in case of water soluble $\mathrm{C}$.

\section{Naso-mucosal site of drug absorption}

Structurally nose is divided in two different nasal cavities by a septum. Each cavity consists of three regions named as vestibule, respiratory region and olfactory region. Functionally, the nasal cavity plays an important protective role to filter, warm and humidify the inhaled air before it reaches the lower airways. It provides a supply and conditioning of air to lungs. Any inhaled particles or microorganisms are trapped by the hair of the nasal vestibule or by the mucus layer covering the respiratory area of the nasal cavity. The mucocilliary clearance mechanism of the mucus layer gradually carries such particulates to the back of the throat, down the esophagus, and further into the gastrointestinal tract. Nasal mucosa also have the metabolic capability of converting endogenous materials into compounds that are eliminated more readily [4]. For drug absorption by nasal mucosa and delivery to brain involves the olfactory neural cells, or axons, are unmyelinated cells and are interspaced between supporting cells. They originate at the olfactory bulb and terminate at the apical surface of the olfactory neuroepithelium. Nanoparticles of sufficiently small size or modified formulations could potentially be transported via axons through the olfactory bulb into the olfactory cortex and from there to the caudal pole of the cerebral hemisphere and into the cerebrum and the cerebellum. Hence, these are all potential delivery sites for nose to brain drug transport route via the olfactory epithelium [5]. Different researchers have published their reports in recent times describing improved or better absorption potential trough nasal mucosal route compared to conventional oral or parenteral routes.

\section{Formulations intended for nasal delivery to target brain}

Different type of formulations have been developed and studied for naso-mucosal drug delivery to brain such as micro or nano emulsion, nasal gel or thermoresponsive nasal gel etc. Some recent reports on nasal emulsion for brain targeted delivery include microemulsion formulation of Rivastigine [6], Palperidone microemulsion [7] or more recent curcumin micro-emulsion by Shinde and Devrajan [8] or nanoemulsion formulations of Quetiapine [9]. All of the articles reported promising drug absorption via nasal route. Among other intra-nasal delivery platforms, gel formulation is very popular in pharmaceutical researchers. Gel formulation can retain on any application site more longer time than solution due to imparted viscosity. For intra nasal application inclusion of bioadhesive polymer helps to retain the dosage form more pronged period of time onto nasal mucosa. There are some polymers that can be converted to gel form by altering $\mathrm{pH}$ or temperature. The list includes synthetic as well as natural polymers [10]. A delivery system which is solution in normal temperature but converted into gel at elevated temperature can also retain longer onto mucosal epithelium as well as enhances patient compliance in term of easy administration, measurable dosage etc. Such kind of system is termed as in situ gellying drug delivery platform. In situ nasal thermoresponsive gel delivery system has been developed with many drugs such as Ropinirole [11], Levadopa [12], Midazolam

${ }^{*}$ Corresonding author: Chatterjee B, Kulliyyyah of Pharmacy, International Islamic University Malaysia, Kuantan 25200, Malayisa; Tel: +60 9-571 6400; E-mail: bdpharmaju@gmail.com

Received February 24, 2017; Accepted February 26, 2017; Published March 05 , 2017

Citation: Chatterjee B (2017) Nose to Brain Drug Delivery: A Recent Update. J Formul Sci Bioavailab 1: 105

Copyright: ( 2017 Chatterjee B. This is an open-access article distributed under the terms of the Creative Commons Attribution License, which permits unrestricted use, distribution, and reproduction in any medium, provided the original author and source are credited. 
[13] etc. This dosage form showed efficient brain targeted drug delivery via intra nasal route. But effect of temperature on such type of dosage form is very crucial and needs critical evaluation [14].

\section{Investigation of drug distribution in brain}

To ensure effective delivery to brain the distribution of drug into brain or central nervous system must be investigated which is quite challenging for pharmaceutical researchers. The drug concentration in cerebro-spinal fluid (CSF) can be easily measured but this is not a measurement of drug crossing blood brain barrier [15]. This measurement cannot be done by in vitro model because cultured endothelial cells over-estimate passive transport across the BBB, and under-estimate carrier-mediated transport across the BBB [16]. By in vivo animal model different approaches have been done to investigate blood brain barrier transport. The drug-receptor occupancy in brain is proportional to the free drug concentration in brain [17]. So measurement of free drug concentration often leads to estimation of drug distribution in brain cell. Brain drug distribution in animal model can be measured by radioactive assay where a radioactive tagging is done with the drug to be estimated. Then the radioactivity present in brain tissue was measured using shielded well-type gamma scintillation counter. From the results direct transport efficiency of the delivered drug can be calculated [7]. Another way to measure drug concentration in brain tissue after targeted delivery is collecting animal brain tissues and analyzing by suitable chromatographic technique (HPLC/ LC-MS) after proper treatment, homogenization and sample preparation [3]. This second technique is relatively easier with respect to availability of instrument set up and regulatory guidelines.

\section{Challenges of intra nasal drug delivery}

Despite improved drug absorption from nasal route to target brain, there are several potential limitations which restrict the success of this route of drug delivery. Irregular drug absorption and less retention time of drug due to mucocilliary clearance is major challenge for delivered drug into the nasal cavity. Normal solution drop cannot stay longer period of time into nasal cavity. We need modified formulation strategy to ensure presence of drug into nasal cavity for longer period of time. Absorption enhancers cannot be used in intra nasal preparation due to possible toxicity. Also there is variation in absorbed drug amount in different regions of brain and spinal cord [18]. Nasal absorption may vary with nasal congestion due to cold or allergic condition. Administration of exact accurate dose by the patient is also a problem in intra-nasal delivery system.

Another major limitation is formulation delivered via nasal route may cause naso-mucosal irritation and toxicity due to the potential incompatibility of many formulation additives with nasal mucosal tissue [19]. Some preservatives in working concentration are also sensitive to nasal mucosa like benzalkonium chloride [20]. Loss of epithelial cell, loss of ciliary layer and shrinkage of mucosal layer are common toxic effects that might be incurred by incompatible active or inactive ingredient of formulation [21]. Repeated uses of nasal drops cause such type of adverse effects on nasal mucosal layer. So all the ingredients used in a formulation must be investigated for nasomucosal toxicity.

\section{Future Scope}

The nose to brain delivery route has potential to become alternative of invasive methods of drug delivery to brain in terms of improved drug absorption and less systemic adverse effect. But the limitations of variable absorption or nasal toxicity must be circumvented in order to enjoy the advantages. Moreover the absorption pathways is not also very clearly demonstrated and established. Therefore there is necessity of more studies to exploit this route extensively and successfully.

\section{References}

1. Engelhardt B, Liebner S (2014) Novel insights into the development and maintenance of the blood-brain barrier. Cell and Tissue Research 3: 1-10.

2. Patel JP, Frey BN (2015) Disruption in the blood-brain barrier: The missing link between brain and body inflammation in bipolar disorder? Neural Plasticity.

3. Rankovic Z (2015) CNS Drug Design: Balancing Physicochemical Properties for Optimal Brain Exposure. Journal of Medicinal Chemistry.

4. Illum $L$ (2003) Nasal drug delivery-possibilities, problems and solutions. Journa of Controlled Release 87: 187-98.

5. Gartziandia O, Egusquiaguirre SP, Bianco J, Pedraz JL, Igartua M, et al. (2016) Nanoparticle transport across in vitro olfactory cell monolayers. International Journal of Pharmaceutics 499: 81-89.

6. Shah BM, Misra M, Shishoo CJ, Padh H (2015) Nose to brain microemulsionbased drug delivery system of rivastigmine: formulation and ex-vivo characterization. Drug Delivery 22: 918-930.

7. Patel MR, Patel RB, Bhatt KK, Patel G, Gaikwad RV (2016) Paliperidone microemulsion for nose-to-brain targeted drug delivery system pharmacodynamic and pharmacokinetic evaluation, Drug Delivery 23: 346-354.

8. Shinde RL, Devarajan PV (2017) Docosahexaenoic acid-mediated, targeted and sustained brain delivery of curcumin microemulsion, Drug Delivery 24: 152-161.

9. Boche M, Pokharkar V (2016) Quetiapine Nanoemulsion for Intranasal Drug Delivery: Evaluation of Brain-Targeting Efficiency. AAPS PharmSciTech.

10. Karavasili C, Fatouros DG (2016) Smart materials: In situ gel-forming systems for nasal delivery. Drug Discovery Today. 8

11. Rao M, Agrawal DK, Shirsath C (2017) Thermoreversible mucoadhesive in situ nasal gel for treatment of Parkinson's disease. Drug Dev and Industrial Pharmacy 43: 1.

12. Sharma S, Lohan, Murthy RSR (2014) Formulation and characterization of intranasal mucoadhesive nanoparticulates and thermo-reversible gel of levodopa for brain delivery. Drug Dev Ind Pharm 40: 869-878.

13. Parhizkar E, Emadi L, Alipour S (2017) Development and evaluation of midazolam in situ nasal gel properties in presence of solubility enhancers at cilia-friendly $\mathrm{pH}$. Macromolecular Research: 1-7.

14. Morsi N, Ghorab D, Refai H, Teba H (2016) Ketoroloac tromethamine loaded nanodispersion incorporated into thermosensitive in situ gel for prolonged ocular delivery. International Journal of Pharmaceutics 506: 57-67.

15. Pardridge WM (2016) CSF, blood-brain barrier, and brain drug delivery. Expert Opinion on Drug Delivery 5247: 1-13

16. Bicker J, Alves G, Fortuna A, Falcão A (2014) Blood-brain barrier models and their relevance for a successful development of CNS drug delivery systems: A review. European Journal of Pharmaceutics and Biopharmaceutics.

17. Loryan I, Sinha V, Mackie C, Peer VA, Drinkenburg WH, et al. (2015) Molecular properties determining unbound intracellular and extracellular brain exposure of CNS drug candidates. Molecular Pharmaceutics: 12: 520-532.

18. Pardeshi CV, Belgamwar VS (2013) Direct nose to brain drug delivery via integrated nerve pathways bypassing the blood-brain barrier: an excellent platform for brain targeting. Expert Opinion of Drug Deliv 10: 957-973.

19. Ozsoy Y, Gungor S, Cevher E (2009) Nasal delivery of high molecular weight drugs. Molecules.

20. Mezger E, Wendler O, Mayr S, Bozzato A (2012) Anaphylactic reaction following administration of nose drops containing benzalkonium chloride. Head \& Face Medicine 8: 29

21. Sood S, Jain K, Gowthamarajan K (2014) Optimization of curcumin nanoemulsion for intranasal delivery using design of experiment and its toxicity assessment. Colloids and Surfaces B: Biointerfaces113: 330-337. 